\title{
Kliniken sehen für 2009 einen enormen Investitionsbedarf Besonderer Nachholbedarf bei Bau- und Sanierungsmaßnahmen
}

Nachdem der Bundestag das Gesetz zum ordnungspolitischen Rahmen der Krankenhausfinanzierung ab 2009 Ende des Jahres beschlossen hat, gibt es zwar endlich eine gewisse Planungssicherheit für die Kliniken. Der Investitionsstau in den Kliniken bleibt damit jedoch offen - und Lösungsmöglichkeiten sind kaum in Sicht.

„In den 2087 Krankenhäusern in Deutschland besteht aufgrund der seit Jahren drastisch zurückgefahrenen öffentlichen Investitionsmittel ein gewaltiger Nachholbedarf“, so kommentierte der Hauptgeschäftsführer der Deutschen Krankenhausgesellschaft (DKG), Georg Baum, eine aktuelle Erhebung des Deutschen Krankenhaus Instituts (DKI) (1).

\section{Investitionsbedarf in Höhe von} gut 7 Milliarden Euro

Demnach könnten die deutschen Kliniken allein in diesem Jahr insgesamt 7,38 Milliarden Euro sinnvoll investieren. Hochgerechnet auf alle Allgemeinkrankenhäuser ab 50 Betten wären sie in der Lage, 3,26 Milliarden Euro an Investitionen aus Pauschalfördermitteln zu tätigen. Der größte Posten dieser Fördermittel würden mit 43\%, also 1,41 Milliarden Euro, in den Bereich der Medizintechnik investiert. Mit großem Abstand folgt dann der Bereich ,Gebäude mit Umbaumaßnahmen' mit 13,1 \%, gefolgt von Investitionen zur Einrichtung und Ausstattung der Behandlungsräume bzw. der Krankenzimmer mit 9,9 bzw. 9,3\%.

Für die Einzelförderung ergibt sich für die Krankenhäuser ein Investitionsbedarf in Höhe von 4,12 Milliarden Euro. Allein für Bau- bzw. Umbaumaßnahmen benötigen die Krankenhäuser nur in diesem Jahr insgesamt 2,6 Milliarden Euro an Einzelfördermitteln. Investitionen in technische Betriebsanlagen wie Aufzüge oder Klimaanlagen würden 0,72 Milliarden Euro kosten.

\section{Woher sollen die Mittel dafür kommen?}

Doch wie sieht es mit einer möglichen Finanzierung solcher Investitionen aus
- vor allem vor dem Hintergrund der derzeit bestehenden Unterfinanzierung der Krankenhäuser. Zwar erhalten die Krankenhäuser im Jahr 2009 nach dem Krankenhausfinanzierungsreformgesetz (KHRG), das der Bundestag Ende Dezember verabschiedet hat, zusätzlich über 3,5 Milliarden Euro von der gesetzlichen Krankenversicherung (GKV). Doch auf die Kliniken kommen im nächsten Jahr auch viele Ausgaben zu. Insgesamt liegt die Kostenlast der Häuser bei mehr als 6,7 Milliarden Euro. Diese setzten sich unter anderem aus den „laufenden Kosten“, den Tarif- und Sachkostensteigerungen sowie den Morbiditätslasten zusammen.

Dazu kommen jedoch noch die zusätzlich zu finanzierenden Stellen im Pflegedienst - hier müssen die Krankenhäuser jetzt zwar „nur noch“ einen Eigenanteil von $10 \%$ aufbringen, den Rest übernehmen die Krankenkassen - oder auch die Preisnachlässe für nicht vereinbarte Mehrleistungen. Darüber hinaus sollen Mehrleistungen künftig nur noch in Höhe der variablen Kosten berücksichtigt werden. Dies wird vor allem die Häuser treffen, die besonders gute Arbeit leisten und deshalb mehr und mehr Patienten mit schweren oder aufwendig zu behandelnden Erkrankungen anziehen.

Mit dem aktuellen Hilfspaket der Bundesregierung für die Tarifsteigerungen und den übrigen ordnungspolitischen Weichenstellungen für die Kliniken sind solche Investitionen wohl auch nicht zu tätigen. Denn ersteres deckt gerade die Hälfte des Anstiegs der Personalkosten. Der Rest von rund 2 Milliarden Euro bleibt ebenso Sache der Krankenhäuser wie der Anstieg der Sachkosten. Dazu kommt, dass sich durch die Streckung der Konvergenzphase um ein weiteres Jahr die konvergenzbedingten Mehreinnahmen bei Krankenhäusern mit Budgetzuwächsen halbieren.

Vielleicht können die Kliniken von dem 2. Paket zur Konjunkturförderung profitieren, das der Koalitionsausschuss der Bundesregierung am 12. Januar 2009 verabschiedet hat und das Investitionen in die Infrastruktur in Höhe von 3,5 Milliarden Euro vorsieht. Zusammen mit dem verpflichtenden Finanzierungsanteil der Länder betragen die Finanzhilfen der öffentlichen Hand damit 4,67 Milliarden Euro. Diese Fördermittel sind jedoch nicht allein für Krankenhäuser gedacht, sondern auch für die kommunale Infrastruktur.

\section{Positiver Einfluss auf die deutsche Wirtschaft}

Die Kliniken jedenfalls sind bereit, kurzfristig erhebliche Investitionen umzusetzen, so die Deutsche Krankenhausgesellschaft. Davon würde auch die deutsche Wirtschaft profitieren - und dabei vor allem mittelständische Unternehmen, die die meisten Aufträge von den Krankenhäuser erhalten würden (2).

sts

\section{Literatur}

1 Deutsches Krankenhausinstitut. Krankenhaus Trends - Erhebung Januar 2009. Kurzfristige Investitionsmaßnahmen der Krankenhäuser im Jahr 2009. www.dkgev.de/ media/file/5470.2009-01-19_Anlage-DKI-Erhebung.pdf

2 Prognos AG. Studie - Makroökonomische Auswirkungen zusätzlicher Investitionen im Krankenhausbereich im Jahr 2009. www. dkgev.de/media/file/5439.2009-01-09_PMAnlage-Summary-Prognos-Studie.pdf

Quellen: Pressemitteilungen „DKG zur DKI-Erhebung ,Kurzfristige Investitionsmaßnehmen der Krankenhäuser im Jahr 2009،“ und „Unterfinanzierung der Krankenhäuser bleibt“", herausgegeben von der Deutschen Krankenhausgesellschaft (DKG), Berlin 